\title{
Conductance quantization in mesoscopic graphene
}

\author{
N. M. R. Peres, ${ }^{1}$ A. H. Castro Neto, ${ }^{2}$ and F. Guinea ${ }^{3}$ \\ ${ }^{1}$ Center of Physics and Departamento de Física, Universidade do Minho, P-4710-057, Braga, Portugal \\ ${ }^{2}$ Department of Physics, Boston University, 590 Commonwealth Avenue, Boston, MA 02215, USA \\ ${ }^{3}$ Instituto de Ciencia de Materiales de Madrid, CSIC, Cantoblanco E28049 Madrid, Spain
}

(Dated: July 16, 2018)

\begin{abstract}
Using a generalized Landauer approach we study the non-linear transport in mesoscopic graphene with zigzag and armchair edges. We find that for clean systems, the low-bias low-temperature conductance, $G$, of an armchair edge system in quantized as $G / \tilde{t}=4 n e^{2} / h$, whereas for a zig-zag edge the quantization changes to $G / \tilde{t}=4(n+1 / 2) e^{2} / h$, where $\tilde{t}$ is the transmission probability and $n$ is an integer. We also study the effects of a non-zero bias, temperature, and magnetic field on the conductance. The magnetic field dependence of the quantization plateaus in these systems is somewhat different from the one found in the two-dimensional electron gas due to a different Landau level quantization.
\end{abstract}

PACS numbers: 71.10.Fd, 73.23.Ad, 72.10.Bg, 72.15.Lh.

\section{INTRODUCTION}

Graphene, a two-dimensional (2D) carbon system on a honeycomb lattice presents many anomalous properties when compared with the well-known 2D electron gas obtained in heterostructures. One of the most striking properties is an exotic integer quantum Hall effect (IQHE) predicted theoretically ${ }^{1,2}$, and measured recently ${ }^{3.4}$. The IQHE shows a Hall conductivity given by: $\sigma_{x y}=2(2 n+1) e^{2} / h$, where $n$ is a positive integer. Interestingly, the electrical properties of the graphene systems can be considered classical, in the sense that the measured conductance of the systems is found to increase with the increase of system width and to decrease with the increase of the system length ${ }^{5}$. This experimental result can be understood as an evidence for the presence of disorder in the measured systems. This is further supported by the difficulty in finding experimental evidence for a fractional quantum Hall effect (FQHE) ${ }^{1.6}$.

In the closely related field of carbon nanotubes, recent experiments showed that the conductance of a single wall carbon nanotube is quantized ${ }^{7}$ and shows Fabry-Perot interference patterns. These results can be explained within a generalized Landauer approach, $S$-matrix theory ${ }^{7.8}$, and nonequilibrium Green's function methods 9.10 . The formulation of the problem was introduced by Lake et al $\stackrel{11}{\Perp}$, after the work of Caroli et al ${ }^{12.13 .14}$. Because carbon nanotubes are essentially wrapped graphene, we expect conductance quantization and Fabry-Perot interference patterns to be also observable in ultra-clean graphene. The quantization and the interference patterns, however, should reflect the different types of edges a graphene sheet has (see Fig. 1).

The importance of zig-zag and armchair edges in graphene sheets has been recognized in electron microscopy ${ }^{15}$. Clearly, these two types of edges produce very different electron microscopy intensity curves. We expect, therefore, that coherent charge transport should be different if measured in systems with different types of edges. As in carbon nanotubes ${ }^{16}$, a simple Landauer approach to determine the quantization of the conductance, $G$, can be used for mesoscopic graphene sheets. The calculations follow the generalization of the Lan- dauer approach introduced earlier by Bagwell and Orlando 17 . This type of approach does not account for a discussion of interference patterns, since it neglects multiple electronic reflection at the contacts ${ }^{7.9}$, and it will be discussed elsewhere ${ }^{18}$.

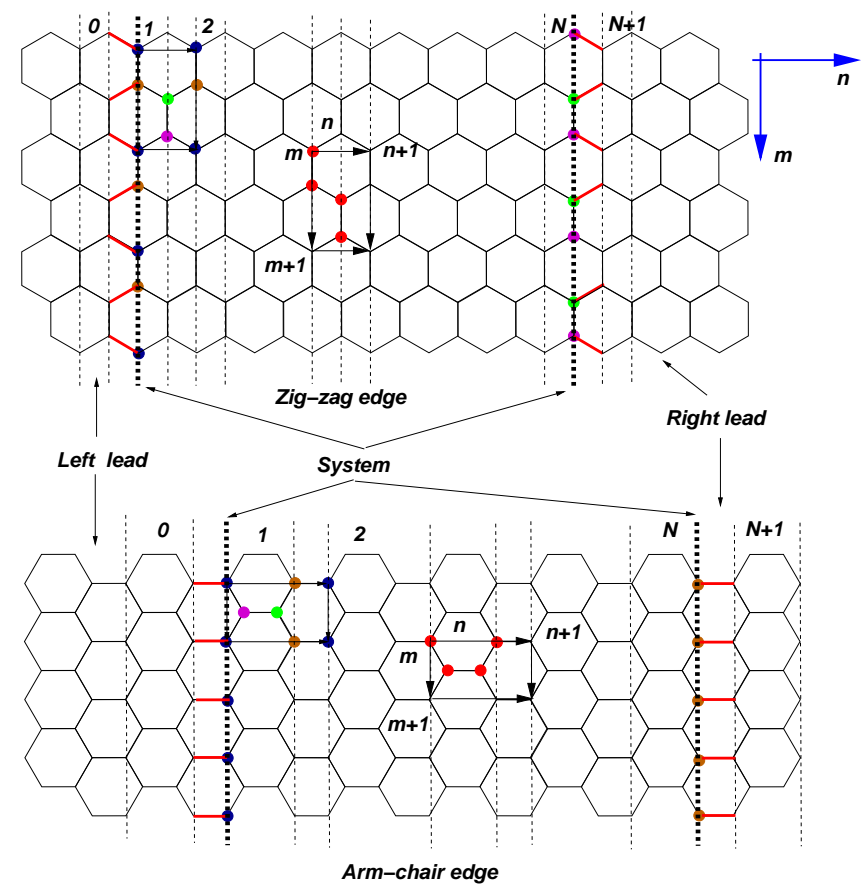

FIG. 1: (color online) Geometry of a finite-size honeycomb lattice. Top: Sample with a zig-zag edge; Bottom: Sample with an armchair edge. The thick dotted lines represent the position of the leads which are assumed to be made out of graphene. The rectangles in the top left corner of the figures (close to the left lead) show the geometry of the unit cell.

This paper is organized as follows: in Sec. [1]we introduce the tight-binding solution for electrons in graphene strips having two different types of edges and the differences and similarities between graphene and carbon nanotubes are discussed. Starting from the tight binding solution of laterally confined electron in graphene strips we introduce a Landauer analysis 
allowing for the calculation of the conductance due to quasione-dimensional transport arising from the confinement; this is done at at zero-temperature and in the zero-bias limit. In Sec. III we discuss the effect of temperature, gate bias, and magnetic field on the conductance curves. It is found that conductance has a minimum value as function of the gate potential (or Fermi energy), from which a "V-like" curve develops, in agreement with measured transport properties in graphene samples. Sec.[V] contains our conclusions.

\section{A LANDAUER ANALYSIS}

The geometry of the problem is shown in Fig. 11 where the left and right leads are supposed to be made of graphene ${ }^{9}$. This aspect is not essential in the approach we develop below, where the contacts to the leads will be modeled by a transmission probability (as done in Refs. [79] in the context of carbon nanotubes). The systems studied here are assumed to have a very asymmetric aspect ratio, where the length, $L$, is much larger than their width, $W$. These systems have some similarity with carbon nanotubes 16 but differ from them in a fundamental way: the absence of periodic boundary conditions along the direction perpendicular to the edges (the $m$ direction). As a consequence, it is possible to have different kinds of strips, characterized by different types of edges. In what follows, we discuss the cases of zig-zag and armchair edges although other edge geometries can be studied with the same methods.

The calculation of the conductance $G$ following a Landauer type of approach ${ }^{16.17}$ requires the solution of a tight-binding problem in a finite geometry. The tight-binding Hamiltonian has the form :

$$
\begin{aligned}
H_{\mathrm{t} . \mathrm{b} .} & =-t \sum_{\langle i, j\rangle, \sigma}\left(a_{i, \sigma}^{\dagger} b_{j, \sigma}+\text { h.c. }\right) \\
& +t^{\prime} \sum_{\langle\langle i, j\rangle\rangle, \sigma}\left(a_{i, \sigma}^{\dagger} a_{j, \sigma}+b_{i, \sigma}^{\dagger} b_{j, \sigma}+\text { h.c. }\right),
\end{aligned}
$$

where $a_{i, \sigma}^{\dagger}\left(a_{i, \sigma}\right)$ creates (annihilates) an electron on site $\mathbf{R}_{i}$, with spin $\sigma(\sigma=\uparrow, \downarrow)$ on sub-lattice $A$ and $b_{i, \sigma}^{\dagger}\left(b_{i, \sigma}\right)$ creates (annihilates) and electron on site $\mathbf{R}_{i}$ with spin $\sigma(\sigma=\uparrow, \downarrow)$ on sub-lattice $B . t$ is the nearest neighbor $(\langle i, j\rangle)$ hopping energy $(t \approx 2.7 \mathrm{eV})$, and $t^{\prime}$ is the next-nearest neighbor $(\langle\langle i, j\rangle\rangle)$ hopping energy $\left(t^{\prime} / t \approx 0.1\right)$. In what follows we suppress the spin index since it plays no role (apart from a degeneracy factor).

In an infinite system the Hamiltonian (1) can be easily diagonalized and one can show that the low energy electronic excitations of the problem reside around the K-points of the Brillouin zone ${ }^{19}$ and have a dispersion given by ( $t^{\prime}$ does not remove the Dirac point):

$$
E_{ \pm}(\mathbf{k})= \pm v_{F}|\mathbf{k}|
$$

where $\mathbf{k}=\left(k_{x}, k_{y}\right)$ is a two-dimensional momentum, and $v_{F}=3 t a /(2 \hbar)$ (where $a$ is the lattice spacing) is the FermiDirac velocity. Eq. (2) describes the dispersion relation of
Dirac electrons with a speed $v_{F}$. One of the consequences of the Dirac dispersion is that the fermions in the system have zero effective mass, and a linearly vanishing density of states, $N(E)(N(E) \propto|E|)$, at low energies. The linearly dispersing electrons and the vanishing of the density of states lead to a very anomalous metallic behavior with many non-Fermi liquid properties $\stackrel{1}{\underline{1}}$. These anomalous properties are reflected in the experimentally measurable quantities, such as the Hall conductivity in the IQHE ${ }^{3.4}$. We are going to show that the presence of Dirac fermions in the spectrum has also a strong effect in the conductance of finite graphene strips.

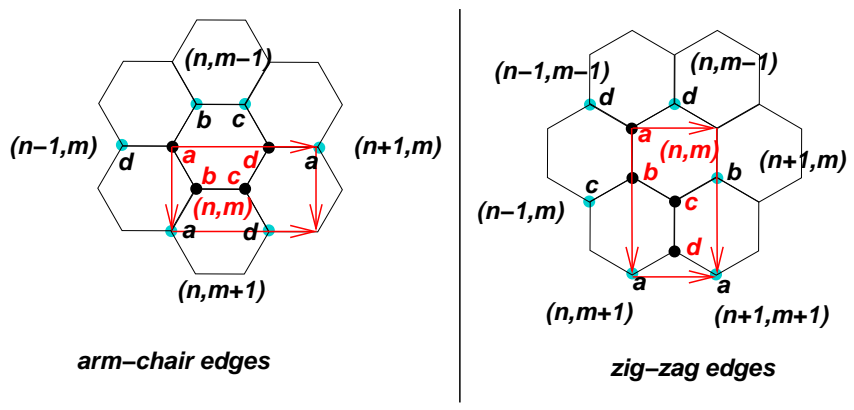

FIG. 2: (color online) Label of the carbon atoms for Hamiltonian 11 in the bulk of the system (only the case $t^{\prime}=0$ is shown for simplicity). The reader is referred to Fig. 11 where the unit cell is shown in each case. The black circles, labeled $a, b, c$, and $d$, inside the rectangle or on the rectangle edges, belong to same unit cell; the other circles represent lattice points in adjacent unit cells connect by the hopping matrix $t$.
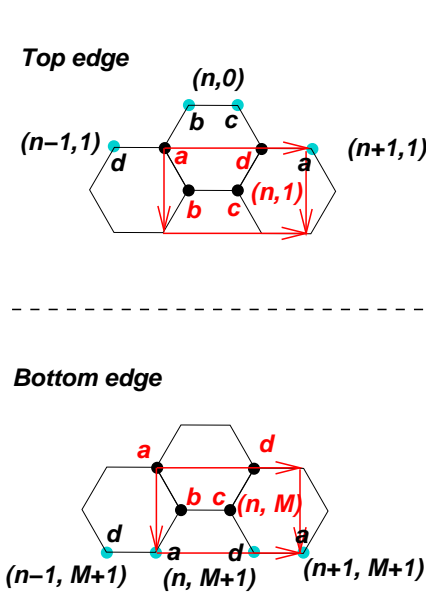

arm-chair edges

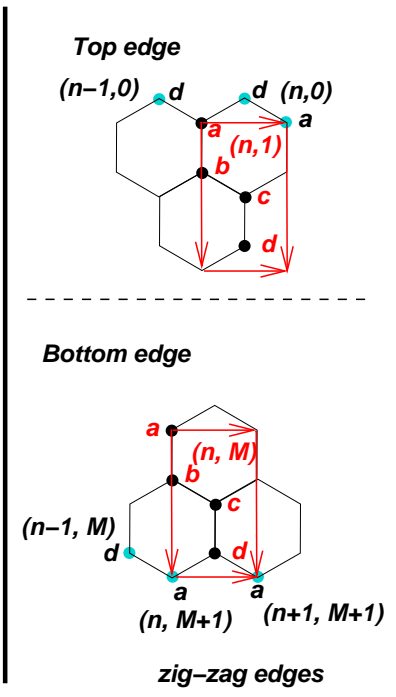

FIG. 3: (color online) This figure shows how to deal with the tightbinding boundary conditions introduced by the free edges of the sample. The cells on the right refer to top free edge and the bottom free edge of a zig-zag sample (top of Fig.11). The cells on the left refer to the top free edge and the bottom free edge of an arm-chair sample (bottom of Fig. 11). As in Fig. 2 only the sites connected by $t$ are shown. 
In a finite system the boundary conditions (and hence, the type of edges) become important in order to define the energy spectrum. In Fig. 2 we establish the labeling of the carbon sites in the bulk of the honeycomb lattice, and in Fig. 3 we present the labels for the carbon atoms close to the free edges of the system. Notice that each unit cell in Fig. 2 and Fig. 3 contains four carbon atoms, labeled by wave function amplitudes $a(n, m), b(n, m), c(n, m)$, and $d(n, m)$, where $n$ and $m$ are integers that label each unit cell. Since we are assuming that in each system only the edges are different, we impose periodic boundary conditions along the direction parallel to the edges (the $n$ direction,) leading to one-dimensional (1D) transport where the electronic states can be labeled by the momentum $q_{x}$. If $N_{y}$ is the number of unit cells along the $m$ direction, then the the tight-binding problem with zig-zag edges has a dimension given by $\left(4 N_{y}+2\right) \times\left(4 N_{y}+2\right)$, while for the armchair edge its dimension is $\left(4 N_{y}+4\right) \times\left(4 N_{y}+4\right)$. For a nanotube, with periodic boundary conditions, the problem can be formulated in terms of two amplitudes instead of four.

The calculation of the conductance of a two-dimensional lattice system with a very asymmetric aspect ratio $\frac{16}{}$ requires the identification of the number of transverse modes, $M(\epsilon)$, at a given energy, $\epsilon . M(\epsilon)$ can be obtained from the solution of the corresponding tight-binding problem. In Fig. [4 we show the energy bands obtained from the diagonalization of the tight-binding Hamiltonian (1) with $t^{\prime}=0$ for the zig-zag and armchair edges. It is clear that the two different types of edges lead to two very different band structures, especially close to zero energy.
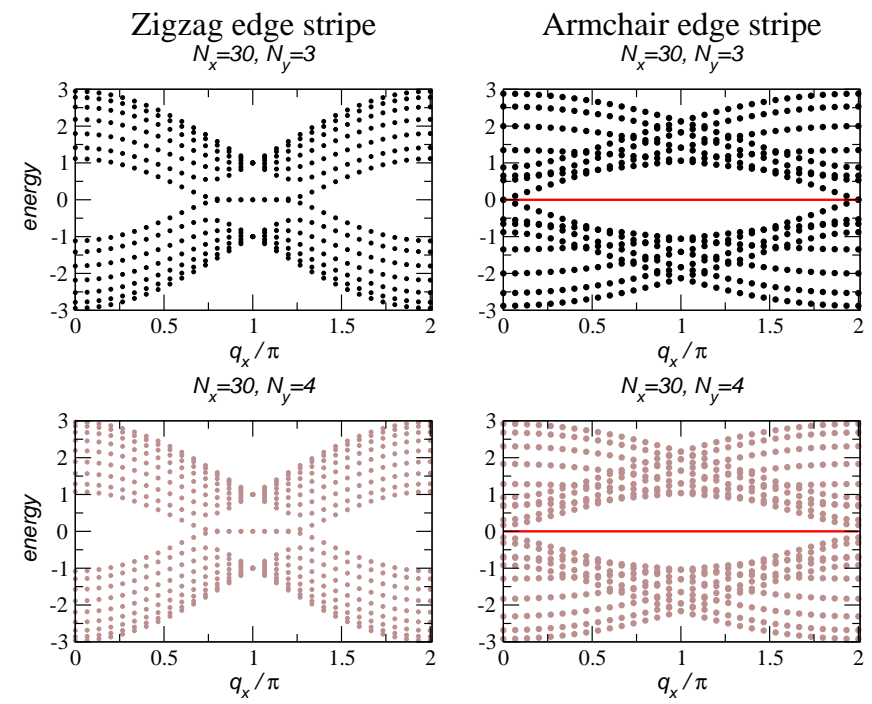

FIG. 4: (color online) Energy levels for $t^{\prime}=0$ (in units of $t$ ) as function of $q_{x}$ (in units of the lattice spacing along the $n$ direction) for the two different types of edges. $N_{y}$ is the number of unit cells perpendicular to the edges (along the $m$ direction) and $N_{x}$ gives the number of momenta used in the abscissa (it also corresponds to the number of unit cells along the $n$ direction). The horizontal line at zero energy on the right panels has an interception with zero energy modes in the upper panel (metallic behavior) and no interception in the lower panel (semi-conducting behavior).
It is important to mention the similarities and the differences between planar systems $\mathrm{s}^{20}$ and carbon nanotubes ${ }^{21}$. An armchair nanotube has the hexagons having two sides perpendicular to the tube axis, in the zig-zag nanotube the hexagons have two sides parallel to the tube axis. A graphene sample with a zig-zag edge has an energy spectrum presenting some similarities with an armchair nanotube. It has two bands crossing the chemical potential at zero energy (and finite momentum). A graphene sample with an armchair edge has a gap at zero energy (near $q_{x}=0$ ) and hence is insulating, except when $N_{y}$, the number of unit cells in the $m$-direction, is a multiple of 3 , in which case the gap goes to zero and the material is metallic (in fact a zero gap semi-conductor; see Fig. $4{ }^{20}$.

A generalized Landauer approach ${ }^{17}$ shows that the tunneling current is given by:

$$
I(V, T)=\frac{2 e}{h} \int d \epsilon M(\epsilon) \tilde{t}(\epsilon, V)\left[f\left(\epsilon-\mu_{1}\right)-f\left(\epsilon-\mu_{2}\right)\right],
$$

where $\tilde{t}(\epsilon, V)$ is the transmission probability per conducting mode at the energy $\epsilon, f(\epsilon)=1 /\left(e^{\epsilon / T}+1\right)$ is the Fermi-Dirac distribution ( $T$ is the temperature and we have put $k_{B}=1$ ), $V$ is the bias voltage applied to the system, and $\mu_{1}\left(\mu_{2}\right)$ is the chemical potential at right (left) lead $\left(\mu_{1}=\mu_{2}+e V\right)$.

In a clean system, all 1D modes can carry electric current, as long as they have a finite velocity in the direction parallel to the edge. Although the zig-zag edge system has zero energy modes with finite $q_{x}$ momentum, the group velocity of these modes is zero when $t^{\prime}=0$, and therefore they do not contribute to the conductance. If we neglect the effect of the next nearest neighbor hopping $\left(t^{\prime}=0\right)$, both edge systems have two conducting zero energy modes (choosing a metallic armchair edge system- $N_{y}$ multiple of three), and as a consequence the small bias conductance is given by $4 e^{2} \tilde{t} / h$, at zero chemical potential.

With the addition of a next nearest neighbor hopping $\left(t^{\prime} \neq\right.$ $0)$ the picture for the zig-zag edge changes substantially: (1) the half-filling case occurs at finite energy (not at zero energy); (2) the flat band states located at zero energy, for $t^{\prime}=0$, become dispersive for $t^{\prime} \neq 0$, and therefore the conductance is modified. Fig. [5 shows the energy levels as function of the momentum $q_{x}$ for a zig-zag system with $t^{\prime}=0$ and $t^{\prime}=0.2 t$, for $N_{y}=3$ (this rather small $N_{y}$ value allows the individual visualization of the transverse modes over the full bandwidth, as it does in Fig. 4 as well).

In order to determine the conductance it is necessary to know how many 1D transverse modes are active for transport at a given energy. For an armchair edge with $N_{y}$ multiple of 3 (and hence a metallic system) one has two conducting modes at zero energy (see Fig. 6) . As one moves away from zero energy the number of modes increases as shown in Fig. 6 Hence, the zero-bias zero-temperature conductance of a clean metallic armchair system is given by:

$$
G=\frac{4 n e^{2}}{h} \tilde{t},
$$

with $n$ a positive integer. The value of $n$ depends on the value of the gate potential controlling the electronic density. The 

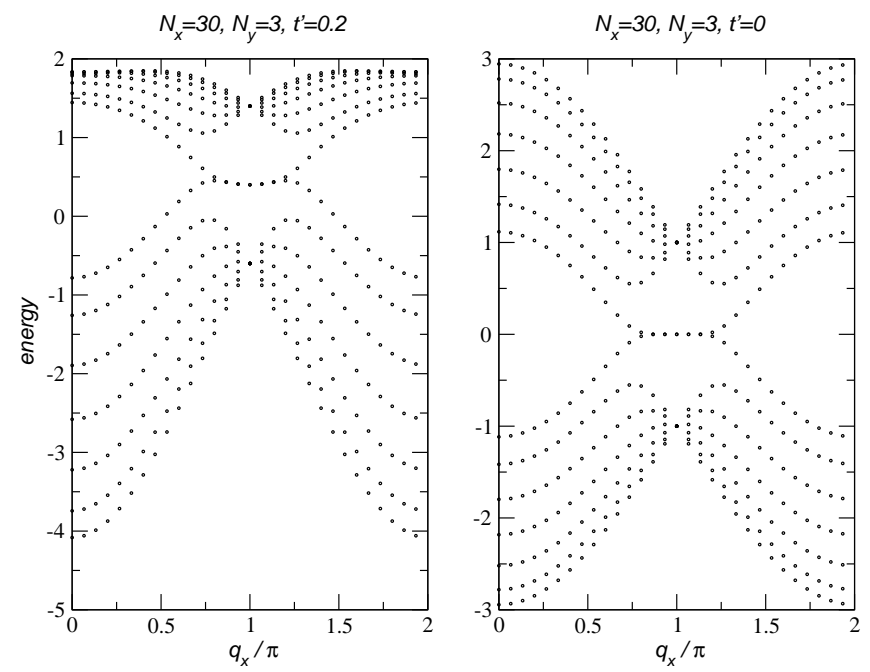

FIG. 5: (color online) Energy levels (in units of $t$ ) as function of $q_{x}$ (in units of the lattice spacing along the $n$ (or $x$ ) direction) for a zig-zag system. Right: $t^{\prime}=0$; left: $t^{\prime}=0.2 t$

conductance also depends on $\tilde{t}$, the transmission probability, assumed to be energy independent for simplicity.

A zig-zag clean system with $t^{\prime}=0$ shows a different dependence. At zero energy $G$ is given by $4 e^{2} \tilde{t} / h$ because of the presence of the two conducting zero-energy modes (see Fig. 6. However, as the gate potential moves slightly away from zero the value of the conductance drops to $2 e^{2} \tilde{t} / h$, since only one transverse mode is available. So the situation with a zig-zag system is somewhat ill-defined. It reasonable to expect that this sudden change on the conductance is difficult to be experimentally observed, since it would require an extreme fine tuning of the experimental parameters. Therefore, we expect that the zero-bias zero-temperature conductance of a system with a zig-zag edge to be given by:

$$
G=\frac{2(2 n+1) e^{2}}{h} \tilde{t},
$$

with $n$ depending also on the value of the gate potential.

The special value of $G$ we found exactly at zero energy (in the zig-zag case) does not survive, however, when electronhole symmetry (by this it is understood that the energy spectrum is not symmetric around zero energy) is broken by the presence of a finite $t^{\prime}$. When $t^{\prime} \neq 0$ the zero mode acquires dispersion and the conductance is given by Eq. (5). In Fig. 7 the effect of $t^{\prime}$ in the low-energy band structure and in $M(\epsilon)$ is visible. Clearly the zero energy modes have been removed, leading to two degenerate dispersive bands, and an asymmetry in energy in the steps of $M$ is introduced. The asymmetry in energy of $M(\epsilon)$ is an experimental way of measuring the value of $t^{\prime}$ in these systems. In the armchair case, the effect of $t^{\prime}$ on $G$ is not as dramatic as in the case of the zig-zag edge system, since in this case the conductance does not have an abrupt change around zero energy.

In a system with several graphene planes each plane contributes to the conductance almost independently because of the weak coupling between graphene sheets. Therefore, the resulting conductance should be, at least approximately, given by the above results multiplied by the number of layers.
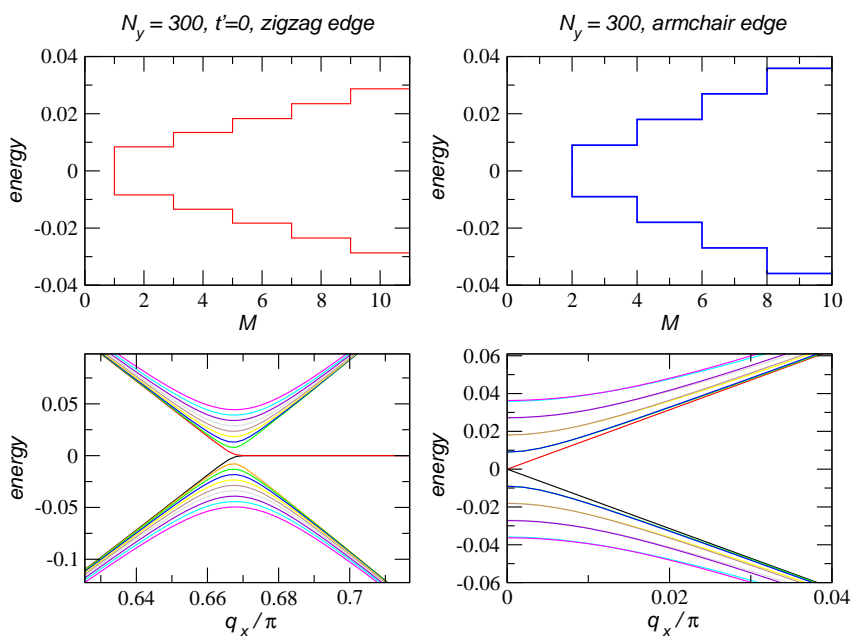

FIG. 6: (color online) Lower panels: one-dimensional energy bands (energy in units of $t$ ) associated with a zig-zag (left) and a armchair edge (right) systems for $t^{\prime}=0$. Upper panels: number of $1 \mathrm{D}$ channels, $M$, as a function of energy (in units of $t$ ).
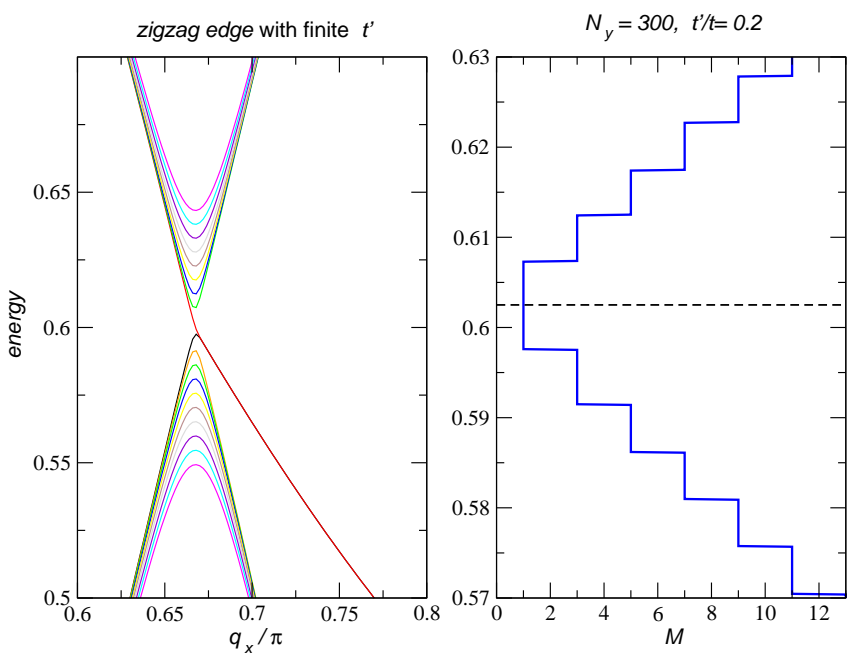

FIG. 7: (color online) Left panel: one-dimensional energy band (energy in units of $t$ ), for a zig-zag edge system with $t^{\prime}=0.2 t$. Right panel: number of $1 \mathrm{D}$ channels, $M$, as a function of energy (in units of $t$ ). The horizontal dashed line helps in stressing the fact that $M(\epsilon)$ is asymmetric as a consequence of a finite $t^{\prime}$.

\section{TEMPERATURE, MAGNETIC FIELD, AND VOLTAGE BIAS EFFECTS.}

In this section we discuss how the zero-bias and zerotemperature results are modified by considering the more gen- 
eral case of a finite bias, temperature, and magnetic field applied perpendicular to the graphene plane.

Close to equilibrium (where $\mu_{1} \simeq \mu_{2}=E_{F}$ ) the conductance can be obtained from 3 as:

$$
G(V \simeq 0, T)=\frac{2 e^{2}}{h} \int d \epsilon M(\epsilon) \tilde{t}(E, V)\left(-\frac{d f\left(\epsilon-E_{F}\right)}{d \epsilon}\right) .
$$

For finite bias the conductance is determined from Eq. (3) after a simple numerical derivative in relative to the bias potential. Notice that in equilibrium, the changes in $E_{F}$ can be obtained by simple changes in the value of the gate voltage. The results for $G(V, T)$ as function of $E_{F}, V$, and $T$, are shown in Fig. 8 One can clearly sees that, as predicted, the conductance is quantized in units of $2 \tilde{t} e^{2} / h$, being even in the case of the armchair edge and odd in the case of the zig-zag edge. The temperature makes the plateaus in the conductance smooth. Application of a bias voltage $V$ shifts the position of the conductance plateaus, as expected.
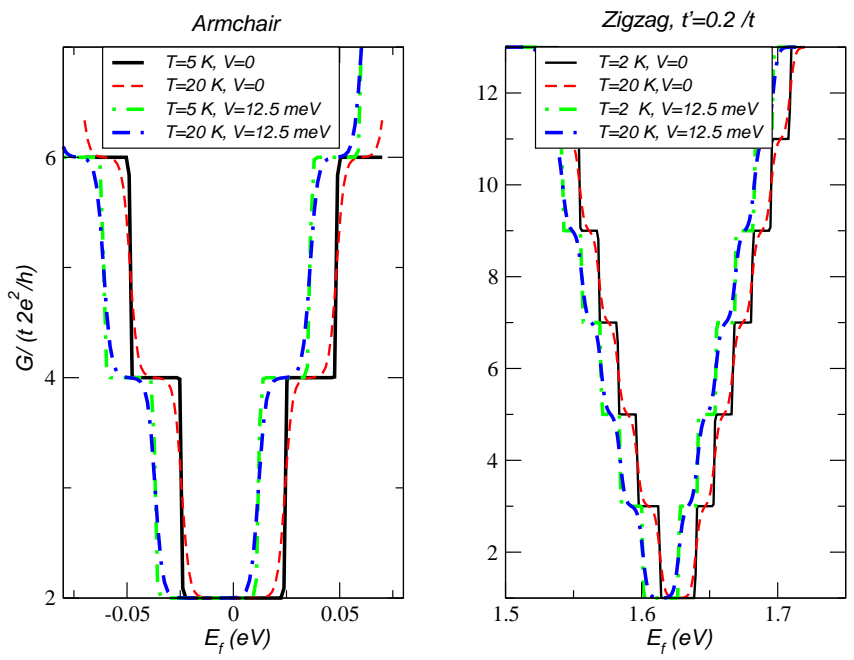

FIG. 8: (color online) Conductance (in units of $2 \tilde{t} e^{2} / h$ ) for armchair and zig-zag systems as function of $E_{F}$, for various values of $T$ and $V(t=2.7 \mathrm{eV})$.

The effect of an external magnetic field in the conductance quantization of the 2D electron gas was experimentally studied in the past ${ }^{23}$ and discussed in general terms by Büttiker ${ }^{24}$. We consider only the case of systems with zig-zag edges for simplicity since, with periodic boundary conditions along the $n$ direction, a unit cell with only two atoms can be chosen. In the presence of an applied perpendicular magnetic field $B$ the hopping integrals change to:

$$
t_{i j} \rightarrow t_{i j} e^{i \theta_{i j}}
$$

where the phases $\theta_{i j}=2 \pi \int_{i}^{j} \boldsymbol{A} \cdot d \boldsymbol{l} / \phi_{0}$, and $\phi_{0}=h / e$ is the quantum of flux. The sum of $\theta_{i j}$ over a close path defined by the hopping integrals must equal the value $2 \pi \phi_{P} / \phi_{0}=$ $2 \pi B A_{P} / \phi_{0}$, where $A_{P}$ is the area enclosed by the path $P$. For the honeycomb lattice, the equation for the amplitudes of the tight-binding Hamiltonian (1) can be written as:

$$
\begin{aligned}
\epsilon a_{n, m} & =-t\left[b_{n, m}+b_{n-1, m}+e^{i 2 \pi \phi m} b_{n, m-1}\right] \\
& -t^{\prime}\left[e^{i \pi \phi / 3} a_{n, m-1}+e^{-i 2 \pi \phi(m-5 / 6)} a_{n+1, m-1}\right. \\
& +e^{-i 2 \pi \phi(m-1 / 6)} a_{n+1, m}+e^{-i \pi \phi / 3} a_{n, m+1} \\
& \left.+e^{i 2 \pi \phi(m+1-5 / 6)} a_{n-1, m+1}+e^{i 2 \pi \phi(m-1 / 6)} a_{n-1, m}\right], \\
\epsilon b_{n, m} & =-t\left[a_{n, m}+a_{n, m+1}+e^{-i 2 \pi \phi m} a_{n+1, m}\right] \\
& -t^{\prime}\left[e^{-i \pi \phi / 3} b_{n, m-1}+e^{-i 2 \pi \phi(m-1 / 6)} b_{n+1, m-1}\right. \\
& +e^{-i 2 \pi \phi(m-5 / 6)} b_{n+1, m}+e^{i \pi \phi / 3} b_{n, m+1} \\
& \left.+b_{n-1, m+1} e^{i 2 \pi \phi(m+1-1 / 6)}+e^{i 2 \pi \phi(m-5 / 6)} b_{n-1, m}\right],
\end{aligned}
$$

where $\phi=B A_{c} / \phi_{0}$, and $A_{c}$ is the area of an hexagon $\left(A_{c}=\right.$ $3 \sqrt{3} a^{2} / 2$ with $a \approx 1.4 \AA$ in graphene). For $\phi=0$ we obtain the results of Sec. II

In the presence of a magnetic field the states of the bulk graphene are described in terms of Landau levels. At low energies, when the Dirac fermion description (2) is valid, the energy levels are given by:

$$
E_{ \pm}(n)=-3 t^{\prime}+\frac{2 \alpha}{\ell_{B}^{2}} n \pm \sqrt{\frac{\alpha^{2}}{\ell_{B}^{4}}+\frac{2 \gamma^{2}}{\ell_{B}^{2}} n},
$$

where we have assumed $t^{\prime} \ll t$, and defined $\ell_{B}=\sqrt{\hbar / e B}$ as the magnetic length, $\alpha=9 t^{\prime} a^{2} / 4$, and $\gamma=3 t a / 2$ $(n=1,2, \ldots)$. For $t^{\prime}=0$, the energy levels are given by: $E_{ \pm}(n)= \pm \sqrt{2} \gamma \ell_{B}^{-1} \sqrt{n}$. This result shows that, for the case of Dirac fermions, and unlike the ordinary 2D electron gas, the Landau levels are not equally spaced ${ }^{22}$. Notice that the cyclotron energy, $\hbar \omega_{c}=\sqrt{2} v_{\mathrm{F}} \hbar / l_{\mathrm{B}}$, is much larger than the Zeeman energy, $g \mu_{B} B\left(g \approx 2, \mu_{B}\right.$ is the Bohr magneton for $B=12 \mathrm{~T}, \hbar \omega_{c} \approx 0.142 \mathrm{eV}$ and $g \mu_{B} B \approx 7 \times 10^{-4} \mathrm{eV}$ ). Thus, we disregard the Zeeman energy in what follows.

In a finite system the energy levels given by (10) are modified by the lattice structure and by the presence of edges. This can be clearly seen in Fig. 9 where we plot the solution of (9) for a graphene strip with a zig-zag edge. Clearly at $B=10 \mathrm{~T}$ the Landau level structure predicted by (10) is seen to develop close to the Dirac point. The effect of the magnetic field on $G$ is two fold: (1) the magnetic field leads to non-dispersive magnetic levels, with a large degeneracy; (2) the energy level spacing is modified giving rise to a piling up of energy levels as one moves away from the Dirac point.

Notice that our discussion is valid for weak magnetic fields and hence does not apply to the quantum Hall regime that was discussed in refs. [12]. We observe that states with values of $q_{x}$ away from the system edges can not contribute to the conductance, since their group velocity is zero. Only those levels having non-zero group velocity can act as $1 \mathrm{D}$ channels for electron transport. The piling up of energy levels has the experimental consequence that the observation of many quantized plateaus becomes difficult. In addition, the increase of the degeneracy of each Landau level with the increase of the field reduces the number of observable plateaus, as in the normal electron $\operatorname{gas}^{23}$. On the one hand, when we compare $M(\epsilon)$ 

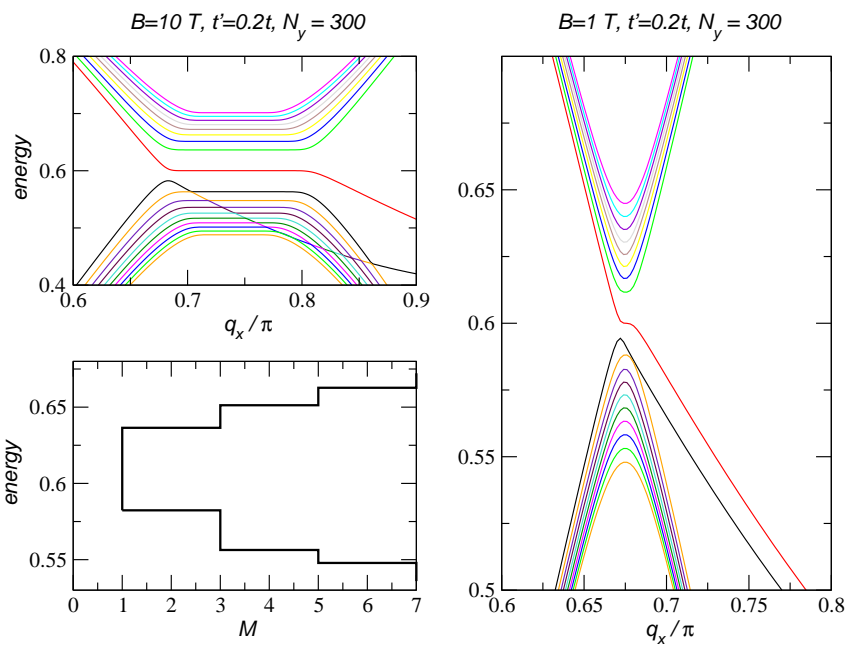

FIG. 9: (color online) Energy spectrum (energy in units of $t$ ) of a graphene strip with a zig-zag edge in a magnetic field $B$ (Left: $B=$ $10 \mathrm{~T}$; Right: $B=1 \mathrm{~T}$ ). Lower left panel: number 1D transverse modes $M$ for $B=10 \mathrm{~T}$.

for $B=0$ and $B=10 \mathrm{~T}$ we see that energy width of the $M=1$ step in the latter case has a much larger value. On the other hand, the piling up of the Landau levels leads to the reduction the energy value of plateaus (see Figs. 7 and 9 . A small field $B$ does not lead the formation of Landau levels, but removes the degeneracy of the band formed from the flat band of zero modes when $t^{\prime}$ is considered, and leads to a $M=1$ energy step width of a larger value when compared with the $B=0$ case.

\section{SUMMARY AND CONCLUDING REMARKS}

We have discussed the tunneling transport in clean mesoscopic graphene strips. We show that different graphene strips have different conductance values due to different types of edges. As a general consequence of the graphene band structure, and at odds to the usual 2D electron gas systems, the conductance always increase as we move away from the Dirac point, and therefore the conductance assumes a "V"-shape form as a function of the gate potential. The lowest value of $G / \tilde{t}$ in the zig-zag edge system is $2 e^{2} / h$ (for a non-zero, albeit small, $\left.t^{\prime}\right)$, whereas in an armchair edge system we find $4 e^{2} / h$.

We have studied in detail the plateaus in the conductance of graphene strips as a function of temperature, applied gate voltage, and external magnetic field. We have seen that the temperature smoothes out the plateaus and that applied gate voltages shift the plateaus in energy. We have also discussed the effect of next nearest neighbor hopping energy $t^{\prime}$, that breaks the particle-hole symmetry of the problem and introduces dispersion for in the zero modes. The effect of a finite magnetic field is quite interesting in these systems because of the unusual relation between the energy and the Landau level index. We show that a magnetic field has effect in piling up the conductance steps and modify their size in energy. These effects should be easily observable in ultra clean mesoscopic graphene strips.

For graphene samples of $10-100 \mu \mathrm{m} \operatorname{size}^{25}$ it was found that the conductivity, given by $\sigma=G L / W$, has the universal value of $\sim 4 e^{2} / h$. This result can be understood using a bulk calculation of the effect of vacancies on the electric linear response (Kubo formula) of Dirac fermions $\stackrel{1}{*}$. ¿From the point of view of coherent tunneling, these experimental results indicate that these samples are in the ohmic regime, having a mean free path shorter than the system size. We believe, however, that in ultra clean graphene samples it will be possible to observe conductance quantization and interference patterns, as it is the case of carbon nanotubes. We hope that our results will stimulate further studies of transport in these amazing systems.

\section{Acknowledgments}

We thank A. Geim, P. Kim, and W. de Heer for stimulating discussions. N.M.R.P. thanks ESF Science Programme INSTANS 2005-2010 and FCT under the grant POCTI/FIS/58133/2004. A.H.C.N. was supported through NSF grant DMR-0343790.
${ }^{1}$ N. M. R. Peres, F. Guinea, and A. H. Castro Neto cond-mat/0506709 and cond-mat/0512091

2 V. P. Gusynin, and S. G. Sharapov, Phys. Rev. Lett. 95, 146801 (2005), and cond-mat/0512157

3 K.S. Novoselov, A.K. Geim, S.V. Morozov, D. Jiang, M.I. Katsnelson, I.V. Grigorieva, S.V. Dubonos, and A.A. Firsov, Nature 438, 197 (2005).

4 Yuanbo Zhang, Yan-Wen Tan, Horst L. Stormer, and Philip Kim, Nature 438, 201 (2005).

5 Claire Berger, Zhimin Song, Tianbo Li, Xuebin Li, Asmerom Y. Ogbazghi, Rui Feng, Zhenting Dai, Alexei N. Marchenkov, Edward H. Conrad, Phillip N. First, and Walt A. de Heer, J. Phys. Chem., 108, 19912 (2004).
${ }^{6}$ A. H. Castro Neto, F. Guinea, and N. M. R. Peres, cond-mat/0509709

7 Wenjie Liang, Marc Bockrath, Dolores Bozovic, Jason H. Hafner, M. Tinkham, and Hongkun Park, Nature 411, 665 (2001).

${ }^{8}$ For a compreensive presentation of the Landauer formulation see Supriyo Datta, Electronic Transport in Mesoscopic Systems, (Cambridge, 1997).

9 S. Krompiewski, J. Martinek, and J. Barnaś, Phys. Rev. B 66, 073412 (2002).

${ }^{10}$ For a review on the non-quilibrium Green's function method see: J. Rammer, and H. Smith, Rev. Mod. Phys. 58, 323 (1986).

11 Roger Lake, Gerhard Klimeck, R. Chris Bowen, and Dejan Jovanovic, J. Appl. Phys. 81,7845 (1997). 
12 C. Caroli, R. Combescot, P. Nozieres, and D. Saint-James, J. Phys. C:Solid St. Phys., 4, 916 (1971); idem, ibidem, 4, 2598 (1971); idem, ibidem, 5, 21 (1972).

13 R. Combescot, J. Phys. C:Solid St. Phys., 4, 2611 (1971).

14 R. Combescot, and G. Schreder, J. Phys. C:Solid St. Phys., 6, 1363 (1973); idem, ibidem, 7, 1318 (1974).

15 Y. Niimi, T. Matsui, H. Kambara, K. Tagami, M. Tsukada, and Hiroshi Fukuyama, Appl. Surf. Sci. 241, 43 (2005).

16 Weidong Tian, and Supriyo Datta, Phys. Rev. B 49, 5097 (1994).

17 Philip F. Bagwell, and Terry P. Orlando, Phys. Rev. B 40, 1456 (1989).

${ }^{18}$ N. M. R. Peres, F. Guinea, and A. H. Castro Neto, in preparation.

${ }^{19}$ P. R. Wallace, Phys. Rev. 47, 622 (1947).
${ }^{20}$ Kyoko Nakada, Mitsutaka Fujita, Gene Dresselhaus, and Mildred S. Dresselhaus, Phys. Rev. B 54, 17954 (1996).

${ }^{21}$ Riichiro Saito, Mitsutaka Fujita, G. Dresselhaus, and M. S. Dresselhaus, Phy. Rev. B 46, 1804 (1992).

${ }^{22}$ M. H. Johnson and B. A. Lippmann, Phys. Rev. 76, 828 (1949); I. I. Rabi, Z. Phys. 49, 507 (1928).

23 B. J. van Wees, L. P. Kouwenhoven, H. van Houten, C. W. J. Beenakker, J. E. Mooij, C. T. Foxon, and J. J. Harris, Phys. Rev. B 38, 3625 (1988).

${ }^{24}$ M. Büttiker, Phys. Rev. B 38, 9375 (1988).

25 K. S. Novoselov, D. Jiang, T. Booth, V.V. Khotkevich, S. M. Morozov, and A. K. Geim, Proc. Natl. Acad. Sci. 102, 10451 (2005). 